\title{
IMPAIRMENT OF ANTIGEN-SPECIFIC CELLULAR IMMUNE RESPONSES UNDER SIMULATED MICROGRAVITY CONDITIONS
}

\author{
K. JAGANNADHA SASTRY,' PRAMOD N. NEHETE, AND CHERYLYN A. SAVARY
} Department of Veterinary Sciences, The University of Texas, M. D. Anderson Cancer Center, Bastrop, Texas 78602 (K. J.S., P. N. N.) and
Department of Surgical Oncology, The University of Texas, M. D. Anderson Cancer Center, Houston, Texas 77030 (C. A. S.)

\begin{abstract}
SUMMARY
Microgravity has been implicated to play a role in the observed immune dysfunction of astronauts and cosmonauts after either short-term or long-term space travel. These reports, together with studies describing increased levels of microorganisms in the space cabin environment suggest potential risk for in-flight incidences of infectious diseases. In order to understand the mechanism underlying these immune defects, it is important to have a ground-based model that would reliably mimic the effects of microgravity on antigen-specific immune function. We tested the utility of the rotating wall vessel (RWV) technology developed at NASA as a model system because in the RWV the culture medium and the cells rotate synchronously with the vessel, thereby creating simulated microgravity conditions. We compared the RWV to the conventional tissue culture flask (T-flask), for culturing immune precursor cells with cytotoxic T lymphocyte (CTL) activity against synthetic viral peptides. We observed a significant loss of antigen-specific CTL activity in RWV cultures, but not in those from the T-flask, irrespective of the peptide immunogen used for inducing the primary immune response in different mouse strains. Loss of CTL activity in RWV cultures coincided with a significant reduction in CD8 ${ }^{+}$cells as well as $\mathrm{CD}_{4}{ }^{+}$cells and DEC205+ dendritic cells, suggesting adverse effects of RWV culturing on both the effector and accessory cells for the loss of antigen-specific CTL function. These results provide a strong parallel to the reported defects in cell-mediated immunity during space travel and strongly support the utility of the RWV technology as an effective ground-based model for identifying key steps in immune cell dysfunction related to microgravity.
\end{abstract}

Key words: synthetic peptides; cell-mediated immunity; cytotoxic T lymphocytes; rotating wall vessel.

\section{INTRODUCTION}

A survey of literature related to the effects of space travel on the human immune system reveal microgravity-associated immune dysfunction (Taylor et al., 1986, 1997; Sonnenfeld et al., 1990; Taylor and Janney, 1992; Konstantinova et al., 1993; Sonnenfeld and Miller, 1993; Taylor, 1993a, 1993b; Gmunder et al., 1994; Cogoli and Cogoli-Greuter, 1997; Cooper and Pellis, 1998). These include: (1) major changes in certain immune cell populations (Taylor et al., 1986; Sonnenfeld et al., 1990), (2) general depression in the ability of lymphocytes to proliferate in response to mitogens (Taylor et al., 1986; Sonnenfeld et al., 1990; Cogoli and Cogoli-Greuter, 1997; Cooper and Pellis, 1998), (3) decreased production and function of certain types of cytokines (Sonnenfeld and Miller, 1993), (4) reduction in NK cell activity (Sonnenfeld et al., 1990), and (5) weakening of delayed-type hypersensitivity responses (Taylor and Janney, 1992; Gmunder et al., 1994). Most of these studies involved in vitro analysis of blood samples collected soon after landing and comparing the results with the preflight values for that particular individual. Systematic in-flight analysis of the mechanism underlying these observed immune defects, particularly the involvement of microgravity, is not always practical due to financial and technical con-

1 To whom correspondence should be addressed at Department of Veterinary Sciences, The University of Texas, M. D. Anderson Cancer Center, Science Park, 650 Cool Water Drive, Bastrop, Texas 78602. E-mail: jsastry@ mdanderson.org straints. It is, therefore, important to adopt a model system that will mimic the microgravity conditions. In this regard, published reports in the literature indicate that the rotating wall vessel (RWV) technology developed at NASA may offer a useful experimental model because of its ability to create microgravity conditions with wellcontrolled process parameters and reduced levels of hydrodynamic stress (Stewards et al., 1992; Tsao et al., 1992; Jessup et al., 1993; Gmunder et al., 1994; Cooper and Pellis, 1998). The RWV is a horizontally rotating, fluid-filled vessel with membrane diffusion gas exchange suitable for culturing either suspension cells or anchorage-dependent cells (Stewards et al., 1992; Tsao et al., 1992; Jessup et al., 1993; Gmunder et al., 1994; Cooper and Pellis, 1998). In the RWV, the culture medium as well as cells (individual cells or aggregates) or tissue fragments rotate synchronously with the vessel, thereby creating simulated microgravity that enable efficient mass transfer of nutrients and wastes under low-shear conditions (Stewards et al., 1992; Tsao et al., 1992; Jessup et al., 1993; Gmunder et al., 1994; Cooper and Pellis, 1998). Cooper and Pellis (1998) recently showed that phytohemagglutinin- (PHA)-activation of $\mathrm{T}$ cells in RWV is suppressed, and that by allowing the activation to take place under nonrotating conditions, for as short as $30 \mathrm{~min}$, the suppression could be reduced. These results suggested that simulated microgravity resulting from rotation is responsible for the defects in cellular immune functions.

We are interested in delineating the mechanism(s) for the observed effects of microgravity on cell-mediated immunity (CMI) to 
TABLE 1

AMINO ACID SEQUENCE OF SYNTHETIC PEPTIDES

\begin{tabular}{lll}
\hline Peptide & \multicolumn{1}{c}{ Source } & \multicolumn{1}{c}{ Amino acid sequence } \\
\hline C30 & HIV & HIGPGRAFYTTKN \\
E31 & HPV & QLLRREVYDFAFRDL \\
B105 & Sendai virus & HGEFAPGNYPALWSYA \\
D57 & LCMV & RPQASGVYMGNLTAQ \\
B106 & Influenza virus & TYQRTRALVTG \\
\hline
\end{tabular}

${ }^{a}$ Peptides used for immunizing mice, restimulation of immune precursor cells grown in either T-flask or RWV, and pulsing target cells in the CTL assays.

defined antigens. While the CMI involves the activities of both helper T cells (TH) and cytotoxic T lymphocytes (CTL), induction of specific CTL responses is especially important in destroying infected cells and for sustained recovery from infection (Luckacher et al., 1984; Bevan, 1989). We have investigated the utility of the RWV system as a ground-based model for simulated microgravity and ex vivo culturing of immune precursor cells generated in mice. For these studies, we employed a novel mouse model developed in our laboratory that uses noninfectious synthetic peptides as model immunogens for in vivo induction of CTL, capable of killing cells expressing antigens from infectious viruses (Sastry et al., 1992, 1994; Nehete et al., 1994, 1995; Casement et al., 1995, 1996; Sarkar et al., 1995).

We conducted a series of experiments, comparing the RWV to the conventional tissue culture flask (T-flask) for ex vivo expansion of CTL precursors that are specific to a variety of peptide antigens generated in different mouse strains. Our studies revealed that while conventional tissue culturing in the T-flasks resulted in a successful expansion of precursor CTL with efficient antigen-specific CTL activity, those cultured in the RWV showed complete abrogation of the cytolytic function. These results strongly suggest the involvement of the simulated microgravity conditions in the RWV for abrogation of immune cell function, and prove the utility of RWV technology as a suitable ground-based model for spaceflight-related studies on immune cell dysfunctions.

\section{Materials AND Methods}

Peptides. A majority of the peptides used as model antigens in the experiments were demonstrated in our previous studies to be efficient for in vivo induction of cognate antigen-specific CTL that are MHC class I restricted and express CD8 ${ }^{+}$phenotype (Sastry et al., 1992, 1994; Nehete et al., 1994, 1995; Casement et al., 1995, 1996; Sarkar et al., 1995). The peptides included those from the V3 region in the envelope protein gpl20 of human immunodeficiency virus type 1 (HIV-1), the E6 and E7 oncoproteins of human papillomavirus type 16 (HPV-16), and the nucleoproteins of influenza virus, Sendai virus, and lymphocytic choriomeningitis virus (LCMV). The amino acid sequences of various peptides used are listed in Table 1. The peptides were synthesized in the institutional core facility using the Merrifield solid phase method (Merrifield, 1963) either on a modified Vega 250 automatic peptide synthesizer or by the "Bag" method as described by Houghten (1985).

Mice. Three different strains of mice purchased from Charles River Laboratories (Wilmington, MA) were used in the experiments: Balb/c expressing H-2d, C57Bl/6 expressing $\mathrm{H}_{-} 2^{\mathrm{b}}$, and a hybrid strain $\mathrm{CB} 6 \mathrm{Fl}$ expressing $\mathrm{H}-2^{\mathrm{b}} \times \mathrm{d}$.

Generation of CTL in mice. We used a protocol developed earlier in our laboratory, for rapid screening of antigenic peptides, for their capacity to prime specific CTL responses in mice (Sastry et al., 1992). Briefly, the mice were immunized in the hind footpad with the peptide $(100 \mu \mathrm{g})$ emulsified in Freund's complete or incomplete adjuvant. After $7 \mathrm{~d}$, the draining popliteal lymph nodes (PLN) were removed and the cells were separated by gentle homogenization. These cells containing precursor CTL were restimulated with the cognate peptide (at $40 \mu \mathrm{g} / \mathrm{ml}$ ) for $5 \mathrm{~d}$ at $37^{\circ} \mathrm{C}$ by culturing in either the conventional T-flasks or the RWV. Two types of RWV (purchased from Synthecon, Houston, TX) were used: the 55-ml slow-turning lateral vessel (STLV) and the $50-\mathrm{ml}$ high-aspect rotating vessel (HARV). The filling of RWV with medium and cells was as described by Cooper and Pellis (1998). After the 5 -d restimulation in the T-flasks or the RWV, the cells were assayed for the specific CTL activity, by employing syngeneic target cell lines incubated with the cognate peptide and using the standard ${ }^{51}$ Cr-release assay as described earlier by Sastry et al. (1992).

Preparation of target cell lines. The target cell lines employed for the experiments, P815 and EL4, were obtained from American Type Culture Collection and express $\mathrm{H}-2^{\mathrm{d}}$ and $\mathrm{H}-2^{\mathrm{b}}$, respectively. The target cells were radiolabeled with [ ${ }^{51} \mathrm{Cr}$ ]-sodium chromate by incubating for $2 \mathrm{~h}$ at $37^{\circ} \mathrm{C}$ and pulsed with the cognate peptide $(50 \mu \mathrm{g} / \mathrm{ml})$ by incubating for $2 \mathrm{~h} \mathrm{at} 37^{\circ} \mathrm{C}$.

CTL assay. The CTL activity of various cultures was determined by the ${ }^{51} \mathrm{Cr}$-release assay as described by Sastry et al. (1992). Briefly, effector cells from T-flask and RWV cultures were mixed with the appropriate ${ }^{51} \mathrm{Cr}$-labeled target cells at different effector to target (E:T) ratios ranging from 100:1 to 10:1. The cultures were set up in triplicate wells of 96-well round-bottom microtiter plates and incubated for $4 \mathrm{~h}$ at $37^{\circ} \mathrm{C}$. At the end of the incubation period, the supernatants $(100 \mu \mathrm{l})$ were harvested and the radioactivity was estimated in a gamma counter (Wallac, Inc., Gaithersburg, MD). The percentage of specific lysis was calculated as: $100 \times$ (experimental lysis spontaneous lysis)/(maximum lysis - spontaneous lysis). Maximum lysis was determined from supernatants of ${ }^{51} \mathrm{Cr}$-labeled target cells that were lysed with $5 \%$ Triton-X100 and the spontaneous lysis from supernatants of cells cultured in the presence of medium without added effector cells.

Flow cytometric analysis. Lymph node cells obtained from immunized mice were analyzed for $5 \mathrm{~d}$, before and after culturing, in either T-flask or RWV, for expression of surface markers for $\mathrm{T}$ cell subsets (CD4 and CD8) and dendritic cells (DC) $(\mathrm{DEC}-205)$ by flow cytometry. Briefly, $1 \times 10^{6}$ cells were incubated for $20 \mathrm{~min}$ at $4^{\circ} \mathrm{C}$ with fluorescein isothiocyanate (FITC)-, or phycoerythrin (PE)-conjugated antibody, reactive against CD4, CD8, or DEC205 (Caltag, Burlingame, CA and Becton-Dickinson, San Jose, CA). Cells labeled with PE- and FITC-conjugated isotype control antibodies were used as control. The labeled cells were washed, fixed with $1 \%$ paraformaldehyde, and analyzed using a FACScan flow cytometer (Becton-Dickinson). Approximately, 30,000-50,000 events were collected in a list mode and analyzed using the FACScan and Cellquest Research Software (Becton-Dickinson). Forward scatter and side scatter gates were set to exclude erythrocytes and debris, and markers were set using appropriate isotype controls to exclude background fluorescence.

Data analysis. The CTL experiments were conducted, with batches of at least five mice for each peptide, and the experiments were repeated at least three times. Data from flow cytometric analyses of various cell types, before and after culturing for $5 \mathrm{~d}$, in T-flask or RWV, were analyzed for statistical significance by a paired $t$-test (one-tailed).

\section{Results}

The major goal of our study was to determine the effects of microgravity on antigen-specific cellular immune responses, by comparing functional activities of CTL precursor cells cultured using the RWV technology and in the conventional T-flask. We employed a novel mouse model developed in our laboratory, which uses noninfectious synthetic peptides for in vivo induction of CTLs capable of killing virus-infected cells (Sastry et al., 1992, 1994; Nehete et al., 1994, 1995; Casement et al., 1995, 1996; Sarkar et al., 1995).

In the first set of experiments, we immunized Balb/c mice (expressing the MHC class I haplotype $\mathrm{H}-2^{\mathrm{d}}$ ) with a synthetic peptide, C30, from the immunodominant V3-loop region of the envelope protein gp120 of the HIV-1 MN strain. Peptides corresponding to the V3 region in the HIV envelope have been shown previously in our laboratory to be capable of inducing efficient CTL response in the mouse model (Sastry et al., 1992, 1994; Nehete et al., 1994, 1995; Casement et al., 1995). Cells obtained from the draining PLN of 


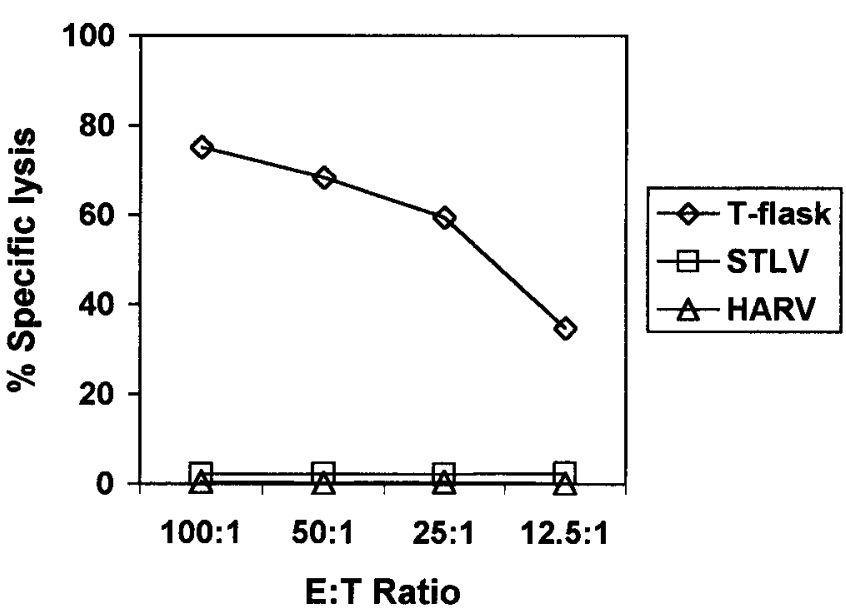

FIG. 1. Culturing immune precursor cells under simulated microgravity conditions in the RWV results in loss of antigen-specific CTL activity. Lymph node cells isolated from Balb/c mice immunized with a synthetic peptide corresponding to the V3 loop region in HIV envelope protein were restimulated in the presence of the peptide in the T-flask, STLV, or HARV for $5 \mathrm{~d}$. The CTL activity in terms of percent specific lysis values against P815 target cells pulsed with the HIV peptide are shown for each of the cultures from T-flask, STLV, and HARV. The control P815 target cells without peptide pulsing showed specific lysis values less than $1 \%$ (not shown). Representative data from at least one of the three separate experiments are shown. See "Materials and Methods" for experimental details.

mice immunized with the C30 peptide were restimulated, for $5 \mathrm{~d}$, in the presence of C30 by culturing in the STLV, the HARV, or in the T-flask, at equal cell numbers and density. The restimulated cells from T-flask cultures showed high levels of C30-specific CTL activity, while those from STLV and HARV showed complete abrogation of the CTL activity (Fig. 1). Similar results showing abrogation of antigen-specific CTL activity in the RWV-cultures were obtained when the experiment was repeated with cells from Balb/c mice immunized with other CTL epitope peptides ( $\mathrm{H}-2^{\mathrm{d}}$-restricted) from LCMV and influenza virus (data not shown). Efforts to recover the CTL function by reculturing the RWV-grown cells in the T-flask did not result in appreciable CTL activity (data not shown). These data, from several experiments employing peptides corresponding to three different viruses, strongly suggest that the abrogation of the CTL function of cultures from the RWV is not related to the peptide antigen employed.

We then sought to verify whether the observed abrogation of CTL activity of the lymph node cells from the Balb/c mice cultured in the RWV is due to the sensitivity of the MHC molecule $\mathrm{H}-2^{\mathrm{d}}$ that presents the peptide antigen to the effector CTL. We performed the experiments using $\mathrm{C} 57 \mathrm{Bl} / 6$ mice which express the $\mathrm{H}-2^{\mathrm{b}}$ haplotype and a peptide termed E31, corresponding to the E6 oncoprotein of HPV-16, which we had demonstrated previously to be capable of inducing CTL in this mouse strain (Sarkar et al., 1995). Restimulation of CTL precursors from lymph nodes of C57Bl/6 mice, immunized with E31, in the RWV resulted in a drastic reduction of the peptide-specific CTL activity compared to those cultured in the T-flask (Fig. 2). Similar results were obtained with cells from $\mathrm{C} 57 \mathrm{Bl} / 6$ mice immunized with another E6 peptide, and two different peptides from the E7 oncoprotein of HPV-16 (data not shown).

We conducted experiments to further investigate whether the antigen (i.e., peptide) or the host MHC (i.e., the H-2 haplotype of the

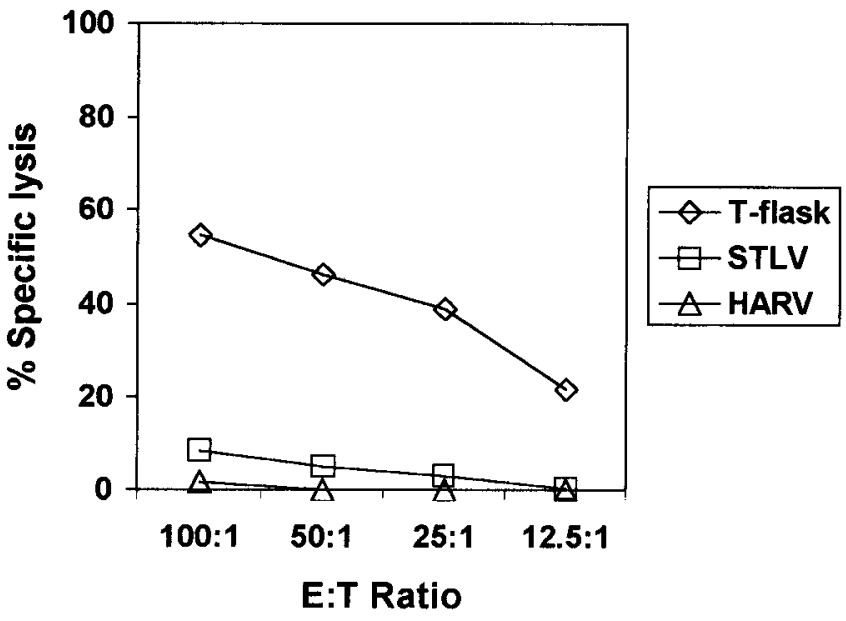

FIG. 2. Loss of antigen-specific CTL activity of immune precursor cells cultured under simulated microgravity conditions in the RWV irrespective of the antigen or the mouse strain used. Lymph node cells isolated from C57Bl/6 mice immunized with a synthetic peptide corresponding to the E6 oncoprotein of HPV-16 were restimulated in the presence of the peptide in the T-flask, STLV, or HARV for $5 \mathrm{~d}$. The CTL activity in terms of percent specific lysis values against EL4 target cells pulsed with the HPV peptide are shown for each of the cultures from T-flask, STLV, and HARV. The control ELA target cells without peptide pulsing showed specific lysis values less than $1 \%$ (not shown). Representative data from at least one of the three separate experiments are shown. See "Materials and Methods" for experimental details.

mouse strain used) would potentially contribute to the observed abrogation of CTL function of cells cultured in the RWV. We immunized a hybrid mouse strain CB6Fl that expresses $\mathrm{H}-2^{\mathrm{b}} \times \mathrm{d}$ with a mixture of five synthetic peptides, corresponding to immunogenic proteins from five different viruses (HIV-1, HPV-16, LCMV, Influenza virus, and Sendai virus). Once again, restimulation of the immune lymph node cells with the peptide mixture in the T-flask resulted in an elaboration of CTL activity specific to each of the peptides, while cells restimulated in the RWV were completely devoid of CTL activity against any of the five peptides used (Fig. 3). Reculturing of cells, obtained after growth in RWV for $5 \mathrm{~d}$, in Tflask did not result in the recovery of antigen-specific CTL activity (data not shown). Together, these results from studies employing a variety of antigenic peptides in mouse strains, expressing two different $\mathrm{H}-2$ haplotypes, clearly indicate that the abrogation of the antigen-specific CTL activity in RWV cultures is not related to either the antigen or its MHC restriction. The most likely reason could be the simulated microgravity conditions in the RWV.

In an attempt to begin understanding the basis for the abrogation of the CTL function of the cultures subjected to growth in the RWV, we first analyzed for differences in the viability of effector and accessory cell populations between the two growth conditions. Cells from three representative experiments, where abrogation of CTL activity was observed in the RWV cultures, but not in T-flask cultures, were selected for viability and flow cytometric analyses of cells expressing CD4, CD8, or DEC-205 surface markers for helper and cytotoxic $\mathrm{T}$ cells, and DC, respectively (Table 2). Overall, RWV cultures showed significantly lower percentages of total viable cell populations than those in the T-flask (41.7\% compared to $21.2 \%$, $P<0.05$ ). Further, comparative analyses of data from three different experiments indicated significant loss of cells expressing all three markers (Table 2). The $\mathrm{CD}^{+}$cells that contribute toward 


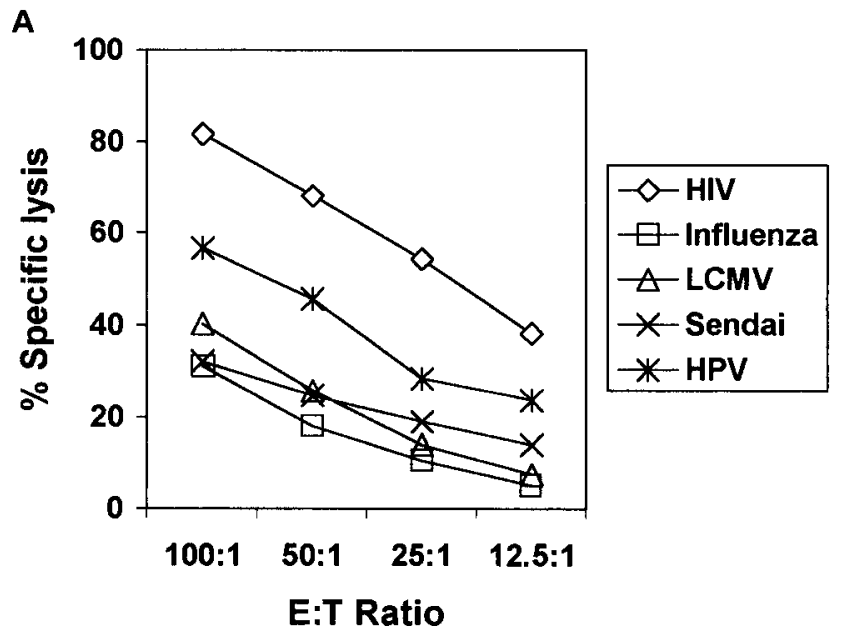

$\mathbf{B}$

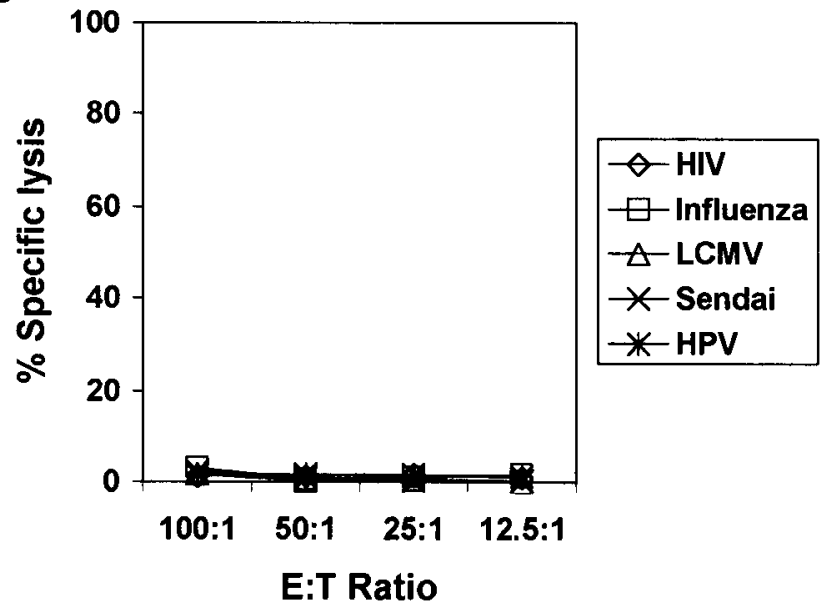

FIG. 3. Simulated microgravity conditions in the RWV result in the loss of CTL activity specific to peptides corresponding to multiple viral pathogens. Lymph node cells isolated from CB6F1 hybrid mice immunized with synthetic peptides corresponding to influenza virus, Sendai virus, HIV, LCMV, and HPV were restimulated in the presence of a mixture of the five cognate peptides in the T-flask and STLV for $5 \mathrm{~d}$. The resulting cultures were assayed for CTL activity specific to each of the five different peptides using P815 target cells or EL4 cells that were pulsed with the peptides. Panel $A$ shows percent specific lysis values for cultures from the T-flask and Panel $B$ shows data for STLV cultures. The specific lysis values for cultures from either Tflask or STLV with unpulsed P815 and EL4 target cells were less than 1\% (not shown). Representative data from at least one of the three separate experiments are shown. See "Materials and Methods" for experimental details.

antigen-specific CTL activity were the most prominently affected population with a change from $51.49 \%$ in the T-flask to $19 \%$ in the RWV $(P=0.0028)$. Significant loss of $\mathrm{CD}^{+}$cells $(P=0.0286)$ involved in helper $\mathrm{T}$ cell function and DEC-205+ cells involved in antigen presentation $(P=0.0386)$ was observed in multiple experiments.

\section{Discussion}

We have investigated the potential utility of the RWV technology developed at NASA as a ground-based model for mimicking simulated microgravity conditions, in order to understand its role in the reported space travel related immune dysfunction, in particular, antigen-specific immunity. While the RWV technology was shown by others to be efficient in supporting the ex vivo culturing of a number of tumor cell types, in our studies, we observed complete abrogation of antigen-specific CTL function of immune precursor cells cultured in the RWV. These results are supported by recent reports describing suppression of $\mathrm{PHA}$ activation of T lymphocytes (Cooper and Pellis, 1998) and failure of IL-2 receptor-mediated signal transduction (Cogoli, 1997; Licato and Grimm, 1999) under simulated microgravity conditions in RWV. Additionally, our results parallel reports in the literature related to effects of space travel on human immune system that implicated microgravity to major changes in certain immune cell populations and depression of cellular immune responses (Taylor et al., 1986, 1997; Sonnenfeld et al., 1990; Taylor and Janney, 1992; Konstantinova et al., 1993; Sonnenfeld and Miller, 1993; Taylor, 1993a, 1993b; Gmunder et al., 1994; Cogoli and Cogoli-Greuter, 1997; Cooper and Pellis, 1998).

In our studies with RWV culturing of the lymph node cells from mice immunized with synthetic peptides, we have analyzed for changes in the viability of various cell types that could potentially be important for the observed abrogation of CTL activity. Consistent with previous reports, (Taylor et al., 1986; Sonnenfeld et al., 1990; Cogoli and Cogoli-Greuter, 1997; Cooper and Pellis, 1998), we observed a significant reduction in the number of viable cells in cultures from the RWV, compared to those from conventional T-flasks. The reduction in populations of $\mathrm{DC}$, and cells expressing $\mathrm{CD}^{+}$or $\mathrm{CD}^{+}$phenotype in repeated experiments was consistent and also statistically significant. The DC is the most potent antigen presenting cell (APC) for both TH and CTL, and plays an important role in the induction of immune response. Any defect in the APC, in terms of the level of cell surface MHC expression, antigen uptake, processing, transport across the endoplasmic reticulum, loading onto nascent MHC molecules, and cell surface presentation could severely compromise the activation of the appropriate effector $\mathrm{T}$ cells. The CD4+ TH cells, on the other hand, upon activation by the antigen, secrete a variety of cytokines that are essential for the adequate functioning of various immune cells, including the CTL, that are important for lysing infected cells. Therefore, any effect of cultural and environmental factors on the viability and/or function of DC, CTL, and TH will adversely affect the outcome of the cellmediated immune responses. In this regard, Sonnenfeld and Miller (1993) reported that spaceflights as well as model systems of spaceflight affected the production and activation of various cytokines including interferons, interleukins, colony stimulating factors, and tumor necrosis factors. However, the implications of such cytokine defects have not been elucidated so far. To date, none of the groundbased studies utilized the RWV technology for addressing the effect of microgravity focused on APC function in relation to antigenspecific immunity. This may be because most studies did not use defined antigens like synthetic viral peptides, as in our experiments, for analyzing specific immune responses such as CTL and TH responses directed against specific viruses. Our results provide a basis for the utility of the RWV technology for determining the possible consequences of simulated microgravity conditions on survival and functions of specialized immune cells, like DC and TH, in terms of antigen presentation and cytokine production that are essential for resistance against infectious agents.

It has been reported that blunting of immune function coincides with a relative increase in infectious microorganisms in the space 
TABLE 2

ANALYSIS OF IMMUNE LYMPH NODE CELLS FROM BALB/C MICE CULTURED FOR 5 D IN EITHER T-FLASK OR RWV

\begin{tabular}{|c|c|c|c|c|}
\hline \multirow[b]{2}{*}{ Growth condition } & \multicolumn{4}{|c|}{ Percent of control ${ }^{a}$} \\
\hline & Total viable cells & $\mathrm{CD}^{+}$cells & $\mathrm{CD8}^{+}$cells & DEC- $205^{+}$cells \\
\hline \multicolumn{5}{|l|}{ T-flask cultures } \\
\hline Experiment 1 & 27.92 & 32.59 & 62.68 & 32.08 \\
\hline Experiment 2 & 53.67 & 57.9 & 41.69 & 59.75 \\
\hline Experiment 3 & 43.64 & 64.00 & 36.21 & 26.15 \\
\hline Mean \pm SD & $41.74 \pm 12.98$ & $51.49 \pm 16.65$ & $46.86 \pm 1.3 .97$ & $39.33 \pm 17.93$ \\
\hline \multicolumn{5}{|l|}{ RWV cultures } \\
\hline Experiment 1 & 18.20 & 16.08 & 38.34 & 12.29 \\
\hline Experiment 2 & 20.53 & 17.80 & 14.06 & 17.60 \\
\hline Experiment 3 & 25.00 & 21.89 & 4.60 & 8.49 \\
\hline Mean \pm SD & $21.24 \pm 3.46$ & $18.59 \pm 2.99$ & $19.00 \pm 17.4$ & $12.8 \pm 4.56$ \\
\hline$P^{\mathrm{b}}=$ & 0.0476 & 0.0286 & 0.0028 & 0.0386 \\
\hline
\end{tabular}

a Controls are numbers of cells at the start of cultures on day 0 .

b Paired $t$-test (1-tailed).

cabin (Taylor et al., 1997). It is, therefore, believed that spaceflightrelated immune dysfunction could have serious consequences on astronauts, in terms of susceptibility to infections and other health problems. However, direct demonstration of microgravity-mediated vulnerability of astronauts to infectious agents, in terms of not being able to mount specific immune responses, is not possible without a ground-based model system that mimics the microgravity conditions. Our results, showing inhibition of antigen-specific CTL activity by culturing the immune precursor cells in the RWV, provide compelling evidence for the microgravity-associated dysfunction of the CMI and provide ground-based confirmatory data for those observed in astronauts and cosmonauts after both short- and longduration space travels. These results form the basis for designing future studies to systematically analyze and understand the effects of microgravity conditions during space travel on human immune system and for developing suitable counter measures.

In addition to understanding the effects of microgravity on immune functions, a desirable outcome of ground-based studies is to be able to devise counter measures that can potentially be tested in spaceflight studies. In this regard, Cooper and Pellis (1998) recently showed that the suppression of phylohernagglutinin- (PHA)activation of $T$ cells in the RWV could be restored by the addition of PMA indicating the possibility of correcting the PKC-mediated signaling pathway, one of the key events in the activation of $\mathrm{T}$ cells. Our results showing the various cell types that may be affected by microgravity will help us not only to understand the mechanistic aspects, but also to address issues related to potential restoration of immune dysfunction resulting from microgravity using the RWV as a model system.

\section{ACKNOWLEDGMENTS}

This work was supported in part by funds from NASA Cooperative Agreement NCC-9-36, National Institute of Allergy and Infectious Diseases AI 42694, and National Cancer Institute CA 65561 and CA77378. All synthetic peptides were prepared in the Synthetic Antigen Core Facility supported by funds from National Institute of Health grant CA 16672.

\section{REFERENCES}

Bevan, M. J. Stimulating killer cells. Nature 342:478-479; 1989.

Casement, K. S.; Arlinghaus, R. B.; Sastry, K. J. Cytotoxic T-lymphocyte response induced by a V3 loop synthetic peptide from an African HIV-l isolate is cross-reactive against HIV-1 strains from North America/Europe regions. AIDS 10:1440-1441; 1996.
Casement, K. S.; Nehete, P. N.; Arlinghaus, R. B., et al. Cross-reactive cytotoxic T lymphocytes induced by V3 loop synthetic peptides from different strains of human immunodeficiency virus type 1 . Virology 211:261-267; 1995.

Cogoli, A. Signal transduction in T lymphocytes in microgravity. Gravit. Space Biol. Bull. 10:5-16; 1997.

Cogoli, A.; Cogoli-Greuter, M. Activation and proliferation of lymphocytes and other mammalian cells in microgravity. Adv. Space Biol. Med. 6:33-79; 1997.

Cooper, D.; Pellis, N. R. Suppressed PHA activation of T lymphocytes in simulated microgravity is restored by direct activation of protein $\mathrm{ki}-$ nase C. J. Leukoc. Biol. 63:550-562; 1998.

Gmunder, F. K.; Konstantinova, I. V.; Cogoli, A., et al. Cellular immunity in cosmonauts during spaceflight on board the orbital MIR station. Aviat. Space Environ. Med. 65:419-423; 1994.

Houghten, R. A. General method for the rapid solid-phase synthesis of large numbers of peptides: specificity of antigen-antibody interaction at the level of individual amino acids. Proc. Natl. Acad. Sci. USA 87:2344$2348 ; 1985$.

Jessup, J. M.; Goodwin, T. J.; Spaulding, G. F. Prospects for use of microgravity-based bioreactors to study three-dimensional host-tumor interactions in human neoplasia. J. Cell. Biochem. 51:290-300; 1993.

Konstantinova, I. V.; Rykova, M. P.; Lesnyak, A. T., et al. Immune changes during long-duration missions. J. Leukoc. Biol. 54:189-201; 1993.

Licato, L. L.; Grimm, E. A. Multiple interleukin-2 signaling pathways differentially regulated by microgravity. Immunopharmacology 44:273$279 ; 1999$.

Luckacher, A. E.; Braciale, V. L.; Braciale, T. J. In vivo effector function of influenza virus-specific cytotoxic T lymphocyte clones is highly specific. J. Exp. Med. 160:814-826; 1984.

Merrifield, R. B. Solid phase peptide synthesis. J. Am. Chem. Soc. 85:21492154; 1963.

Nehete, P. N.; Arlinghaus, R. B.; Sastry, K. J. Use of helper T cell-inducing peptides from conserved regions in HIV-1 env in a non-covalent mixture with a CTL-inducing V3-loop peptide for in vivo induction of long-lasting systemic CTL response. Viral Immunol. 7:189-197; 1994.

Nehete, P. N.; Casement, K. S.; Arlinghaus, R. B., et al. Studies on in vivo induction of HIV-1 envelope-specific cytotoxic T lymphocytes by synthetic peptides from the V3 loop region of HIV-1 IIIB gp120. Cell. Immunol. 160:217-223; 1995.

Sarkar, A. S.; Tortolero-Luna, G.; Nehete, P. N., et al. Studies on in vivo induction of cytotoxic $\mathrm{T}$ lymphocyte responses by synthetic peptides from E6 and E7 oncoproteins of human papillomavirus type 16. Viral Immunol. 8:165 -174; 1995.

Sastry, K. J.; Bender, B. S.; Bell, W., et al. Effects of influenza virus-specific cytotoxic T-lymphocyte responses induced by a synthetic nucleoprotein peptide on the survival of mice challenged with: a lethal dose of virus. Vaccine 12:1281-1287; 1994. 
Sastry, K. J.; Nehete, P. N.; Venkatnarayanan, S., et al. Rapid in vivo induction of HIV-specific CD8* cytotoxic $\mathrm{T}$ lymphocytes by a 15 -amino acid unmodified free peptide from the immunodominant V3-loop of go120. Virology 188:502-509; 1992.

Sonnenfeld, G.; Mandeel, A. D.; Konstantinova, I. V., et al. Effects of space flight on levels and activity of immune cells. Aviat. Space Environ. Med. 61:648-653; 1990.

Sonnenfeld, G.; Miller, E. S. The role of cytokines in immune changes induced by spaceflight. J. Leukoc. Biol. 54:253-258; 1993.

Stewards, R. P.; Goodwin, T. J.; Wolf, D. A. Cell culture for three-dimensional modeling in rotating-wall vessels: an application in microgravity. $\mathrm{J}$. Tissue Cult. Methods 14:51-58; 1992.

Taylor, G. R. Overview of spaceflight immunology studies. J. Leukoc. Biol. 54:179-188; 1993a.
Taylor, G. R. Immune changes during short-duration missions. J. Leukoc. Biol. 54:202-208; 1993b.

Taylor, G. R.; Janney, R. P. In vivo testing confirms a blunting of the human cell-mediated immune mechanisms during space flight. J. Leukoc. Biol. 51:129-132; 1992.

Taylor, G. R.; Konstantinova, I. V.; Sonnenfeld, G., et al. Changes in the immune system during and after spaceflight. Adv. Space Biol. Med. 6:1-32; 1997.

Taylor, G. R.; Neale, L. S.; Dardano, F. R. Immunological analyses of U.S. space shuttle crewmembers. Aviat. Space Environ. Med. 57:213-217; 1986.

Tsao, Y. D.; Goodwin, T. J.; Wolf, D. A., et al. Responses of gravity level variations in the NASA/JSC bioreactor system. Physiologist 35:S49S50; 1992. 